\title{
Pauli algebraic forms of normal and nonnormal operators
}

\author{
Tiberiu Tudor \\ Faculty of Physics, University of Bucharest, P.O. Box MG-11, 077125 Bucharest-Magurele, Romania
}

Aurelian Gheondea

Department of Mathematics, Bilkent University, 06800 Bilkent, Ankara, Turkey, and Institute of Mathematics of Romanian Academy, P.O. Box. 1-764, 014700 Bucharest, Romania

Received March 17, 2006; revised June 18, 2006; accepted July 3, 2006; posted July 27, 2006 (Doc. ID 69093); published December 13, 2006

A unified treatment of the Pauli algebraic forms of the linear operators defined on a unitary linear space of two dimensions over the field of complex numbers $\mathbf{C}^{1}$ is given. The Pauli expansions of the normal and nonnormal operators, unitary and Hermitian operators, orthogonal projectors, and symmetries are deduced in this frame. A geometrical interpretation of these Pauli algebraical results is given. With each operator, one can associate a generally complex vector, its Pauli axis. This is a natural generalization of the well-known Poincaré axis of some normal operators. A geometric criterion of distinction between the normal and nonnormal operators by means of this vector is established. The results are exemplified by the Pauli representations of the normal and nonnormal operators corresponding to some widespread composite polarization devices. (c) 2006 Optical Society of America

OCIS codes: $260.5430,000.3860$.

\section{INTRODUCTION}

In the Pauli algebraic theory of the polarization device operators, it is common knowledge that an ideal general polarizer has the representation ${ }^{1-3}$

$$
\mathrm{P}(\mathbf{n})=\frac{1}{2}\left(\sigma_{0}+\mathbf{n} \cdot \boldsymbol{\sigma}\right)
$$

whereas the representation of a general retarder is ${ }^{1-4}$ :

$$
\mathrm{R}(\mathbf{n}, \delta)=e^{\mathrm{i}(\delta / 2) \mathbf{n} \cdot \boldsymbol{\sigma}}=\sigma_{0} \cos \frac{\delta}{2}+i \mathbf{n} \cdot \boldsymbol{\sigma} \sin \frac{\delta}{2} .
$$

Here $\mathbf{n}$ is a three-dimensional real unit vector and $\boldsymbol{\sigma}$ $=\left(\sigma_{1}, \sigma_{2}, \sigma_{3}\right)$, with $\sigma_{i}$ being the Pauli matrices:

$$
\begin{gathered}
\sigma_{0}=\left[\begin{array}{ll}
1 & 0 \\
0 & 1
\end{array}\right], \quad \sigma_{1}=\left[\begin{array}{ll}
0 & 1 \\
1 & 0
\end{array}\right], \quad \sigma_{2}=\left[\begin{array}{cc}
0 & -i \\
i & 0
\end{array}\right], \\
\sigma_{3}=\left[\begin{array}{cc}
1 & 0 \\
0 & -1
\end{array}\right] .
\end{gathered}
$$

Many concrete problems in the field of light polarization are solved by a Pauli algebraic approach starting with these formulas. ${ }^{1-8}$ But, more generally, the Pauli algebra is a powerful and widespread tool in handling the problems of any two-states quantum systems. ${ }^{9-11}$ The Pauli algebraic approach to these systems is a fundamental one, because it addresses directly the actual topology of their Hilbert state space, which is isomorphic with that of the Poincaré-Bloch sphere. ${ }^{12,13}$
From a mathematical viewpoint, formulas (1) and (2) represent the Pauli algebraic expansions of some very particular kinds of normal operators defined on a unitary linear space of two dimensions over the field of complex numbers $\mathbf{C}^{1}$, namely, orthogonal projectors of rank one and unitary operators, respectively.

In recent times the nonnormal operators have come to light, in matrix forms, in several areas of physics, ${ }^{14-16}$ in connection with a large variety of problems: mode degeneracies for unstable lasers, ${ }^{17}$ light propagation in biaxial absorbing and chiral crystals, ${ }^{18}$ diffraction of atomic beams by "crystals of light," non-Hermitian "nonphysics" of a pile of plates, ${ }^{1}$ phase transition in open quantum systems, ${ }^{20}$ level or resonance crossings and anticrossings, ${ }^{21-24}$ etc.

Referring to the field of light polarization, the operators of all the basic (canonical) polarization devices (homogeneous polarizers and retarders) are normal operators. ${ }^{25}$ On the other hand, the operators of the composite (multilayer) polarization devices may be normal as well as nonnormal, ${ }^{26,27}$ The nonnormal (non-Hermitian) polarizers play an important role in connection with the theory of the generalized quantum measurement. ${ }^{28-31}$

We have to stress that the Pauli algebraic expansions of the orthogonal projectors and unitary operators [Eqs. (1) and (2)] are two isolated formulas that were established inductively, on experimental ground, in the field of polarization theory. ${ }^{32}$ To the best of our knowledge, no systematic theory of the Pauli algebraic forms of the various kinds of operators was elaborated upon until now.

Bearing in mind the above-mentioned enlargement of 
the class of operators implied in solving new problems of the two-state quantum systems, it seems that it is the right time to give a coherent theory of the Pauli forms of the operators on two-dimensional unitary space.

The aim of this paper is to give a unified Pauli algebraic treatment of the linear operators defined on a unitary space of two dimensions over the class of complex numbers $\mathbf{C}^{1}$. In this framework we shall fill the gap between formulas (1) and (2) by establishing the hierarchy of Pauli expansions of the operators of this class, and by integrating deductively Eqs. (1) and (2) of the orthogonal projectors and unitary operators in this hierarchy.

A general result we obtain is that the Pauli algebra provides a remarkable criterion of distinction between the normal and nonnormal operators: in this approach, to each operator on a complex vector space of two dimensions there corresponds a vector in $\mathbf{C}^{3}$, which we shall call the Pauli axis of the operator. The operator is normal if and only if its Pauli axis is a real vector or is reducible to a real vector by a phase shift. The Pauli axes of nonnormal operators are irreducible complex vectors.

Finally we exemplify our general results by deducing the Pauli expansions of the operators of some widespread orthogonal and nonorthogonal composite polarization devices.

\section{NORMAL OPERATORS}

We shall consider a linear operator $A \in L(V)$, where $V$ is a linear space of two dimensions over the field of complex numbers $\mathbf{C}^{1} . \mathrm{L}(V)$ is identified with the algebra of $2 \times 2$ matrices with complex entries. It is well known that the $\sigma$ matrices constitute a basis in the vector space of these operators, so that any such operator may be expressed in the form

$$
\mathrm{A}=a_{0} \sigma_{0}+\mathbf{a} \cdot \boldsymbol{\sigma},
$$

where $a_{0}$ is a generally complex scalar, and $\mathbf{a}$ is a generally complex three-dimensional vector. For reasons that will become evident later on we shall denominate the vector a the axis of the operator. The coefficients $a_{0}, a_{1}, a_{2}$, and $a_{3}$, the components of $\mathbf{a}$, are known in the particular case of Hermitian operators, in polarization optics, under the name of Stokes coefficients (parameters), in which case they are real. We will extend this denomination for any $(2 \times 2$ matrix $)$ operator.

If we label by $A^{\dagger}$ the adjoint of $A$ :

$$
\mathrm{A}^{\dagger}=a_{0}^{*} \sigma_{0}+\mathbf{a}^{*} \cdot \boldsymbol{\sigma}
$$

the condition of normality of $A$ is

$$
\mathrm{AA}^{\dagger}=\mathrm{A}^{\dagger} \mathrm{A} \text {. }
$$

By using Dirac's equation concerning the Pauli expansion of the product of two operators A [Eq. (4)] and B,

$$
\mathrm{B}=b_{0} \sigma+\mathbf{b} \cdot \boldsymbol{\sigma},
$$

namely (e.g., Ref. 2):

$$
\mathrm{AB}=\left(a_{0} b_{0}+\mathbf{a} \cdot \mathbf{b}\right) \sigma_{0}+\left(b_{0} \mathbf{a}+a_{0} \mathbf{b}\right) \cdot \boldsymbol{\sigma}+i(\mathbf{a} \times \mathbf{b}) \cdot \boldsymbol{\sigma} .
$$

Equation (6) takes on the form

$$
\begin{aligned}
& \left(a_{0} a_{0}^{*}+\mathbf{a} \cdot \mathbf{a}^{*}\right) \sigma_{0}+\left(a_{0}^{*} \mathbf{a}+a_{0} \mathbf{a}^{*}\right) \cdot \boldsymbol{\sigma}+i\left(\mathbf{a} \times \mathbf{a}^{*}\right) \cdot \boldsymbol{\sigma} \\
& \quad=\left(a_{0}^{*} a_{0}+\mathbf{a}^{*} \cdot \mathbf{a}\right) \sigma_{0}+\left(a_{0} \mathbf{a}^{*}+a_{0}^{*} \mathbf{a}\right) \cdot \boldsymbol{\sigma}+i\left(\mathbf{a}^{*} \times \mathbf{a}\right) \cdot \boldsymbol{\sigma} .
\end{aligned}
$$

Bearing in mind the anticommutativity of the outer product of two vectors, Eq. (9) leads to

$$
\mathbf{a} \times \mathbf{a}^{*}=0 \text {. }
$$

As a consequence, the two complex-conjugate vectors a and $\mathbf{a}^{*}$ must be collinear, i.e.,

$$
\mathbf{a}^{*}=\lambda \mathbf{a}
$$

where $\lambda$ is a complex number of modulus 1 .

Condition (11) means that, apart from a complex factor, the Pauli axis of a normal operator reduces to a real vector:

$$
\mathbf{a}=e^{\mathrm{i} \alpha} \mathbf{r} .
$$

Hence the Pauli expansion of a normal operator is

$$
\mathrm{A}=e^{\mathrm{i} \alpha_{0}}\left|a_{0}\right| \sigma_{0}+e^{\mathrm{i} \alpha} \mathbf{r} \cdot \boldsymbol{\sigma},
$$

where $\mathbf{r}$ is some real vector, $\alpha_{0}$ is a real scalar modulo $2 \pi$, and $\alpha$ is a real scalar modulo $\pi$.

Obviously, the adjoint of operator $A$ is

$$
\mathrm{A}^{\dagger}=e^{-\mathrm{i} \alpha_{0}}\left|a_{0}\right| \sigma_{0}+e^{-\mathrm{i} \alpha} \mathbf{r} \cdot \boldsymbol{\sigma} .
$$

Finally we shall note that for a normal operator

$$
\mathrm{AA}^{\dagger}=\mathrm{A}^{\dagger} \mathrm{A}=\left(\left|a_{0}\right|^{2}+\|\mathbf{r}\|^{2}\right) \sigma_{0}+2\left|a_{0}\right| \mathbf{r} \cdot \boldsymbol{\sigma} \cos \left(\alpha-\alpha_{0}\right),
$$

where we have made use of Eqs. (13), (14), and (10).

Let us deduce now from the general Pauli expansion of a normal operator [Eq. (13)] some particular forms, corresponding to the most widespread kinds of normal operators.

\section{A. Unitary Operators}

For a unitary operator we have

$$
\mathrm{UU}^{\dagger}=\mathrm{I} \equiv \sigma_{0},
$$

and with Eq. (15) we get two simultaneous equations:

$$
\begin{gathered}
\left|a_{0}\right| \mathbf{r} \cos \left(\alpha-\alpha_{0}\right)=0, \\
\left|a_{0}\right|^{2}+\|\mathbf{r}\|^{2}=1 .
\end{gathered}
$$

If $a_{0} \neq 0$, from Eq. (17) we get:

$$
\alpha-\alpha_{0}=\pi / 2 \text { modulo } \pi
$$

and, on the other hand, Eq. (18) may be fulfilled if we put:

$$
\left|a_{0}\right|=\cos \delta / 2, \quad \mathbf{r}=\mathbf{n} \sin \delta / 2,
$$

with $\mathbf{n}$ a real unit vector.

Coming back to Eq. (13) with Eqs. (19) and (20) we obtain the most general Pauli algebraic form of the unitary operators:

$$
\bigcup=e^{\mathrm{i} \alpha_{0}}\left(\sigma_{0} \cos \delta / 2+\mathbf{i n} \cdot \boldsymbol{\sigma} \sin \delta / 2\right)=e^{\mathrm{i} \alpha_{0}} e^{\mathrm{i}(\delta / 2) \mathbf{n} \cdot \boldsymbol{\sigma}} .
$$


From Eq. (18), if $a_{0}=0$, then $\mathbf{r}=\mathbf{n}$, a unit vector, and the particular case of Eq. (21) is obtained, namely a symmetry (see Subsection 2.D). Equation (21) gives Eq. (2) up to a phase factor.

In polarization optics, such an operator corresponds to a rotation of angle $\delta$ of the polarization state on the Poincare sphere, around an axis defined by the unit vector $\mathbf{n}$, which can be obviously named the Poincaré axis of the operator. For a unitary operator its axis is a real unit vector $\mathbf{n}$, figurable in the real three-dimensional space. It is natural to extend the term of axis of the operator (the Pauli axis of the operator) for the generally complex vector $\mathbf{a}$ in Eq. (4).

In these terms, the remarkable difference between the normal and the nonnormal operators is that the axes of the normal operators are, apart from a phase factor, real vectors, whereas the axes of the nonnormal operators are complex vectors.

If we refer specifically to the optical polarization devices, the operators of the retarders are unitary operators, they pertain to the $\mathrm{SU}(2)$ group, which is isomorphic with $\mathrm{O}(3)$. Therefore an intuitive geometrical representation in $\mathrm{R}^{3}$ for them may be given: a real axis, its Poincaré axis, can be associated to each retarder, and the action of the retarder on the incident light can be represented as a rotation around this axis in $\mathrm{R}^{3}$, more precisely on the Poincaré sphere. Generally, the operators of the (nondepolarizing) optical devices pertain to the six-parameter $\mathrm{SL}(2, \mathrm{c})$ group. For grasping a geometrical insight into the action of such an operator, our intuition has to transcend in a six-dimensional real space, or equivalently in a threedimensional complex space.

\section{B. Hermitian Operators}

For a Hermitian operator,

$$
\mathrm{H}=\mathrm{H}^{\dagger}
$$

with Eqs. (13) and (14) and bearing in mind that $\mathbf{r}$ is a real vector, we get $2 \alpha_{0}=0$, modulo $2 \pi$, i.e.,

$$
\left.\alpha_{0}=0, \pi \text { (modulo } 2 \pi\right),
$$

and $2 \alpha=0$ modulo $\pi$, i.e.,

$$
\alpha=0 \text { (modulo } \pi) \text {. }
$$

Hence for a Hermitian operator all the Stokes coefficients are real:

$$
\mathrm{H}= \pm\left|a_{0}\right| \sigma_{0}+\mathbf{r} \cdot \boldsymbol{\sigma}
$$

\section{Orthogonal Projectors}

A special kind of Hermitian operators are the orthogonal projectors. A projector is idempotent:

$$
\mathrm{P}^{2}=\mathrm{P} \text {. }
$$

With $\mathrm{P}$ given by Eq. (25) and making use of Dirac's equation

$$
(\mathbf{a} \cdot \boldsymbol{\sigma})(\mathbf{b} \cdot \boldsymbol{\sigma})=(\mathbf{a} \cdot \mathbf{b})+i(\mathbf{a} \times \mathbf{b}) \cdot \boldsymbol{\sigma},
$$

for $\mathbf{a}=\mathbf{b}=\mathbf{r}$, Eq. (26) becomes

$$
\left(\left|a_{0}\right|^{2}+\|\mathbf{r}\|^{2}\right) \sigma_{0}+2\left|a_{0}\right| \mathbf{r} \cdot \boldsymbol{\sigma}= \pm\left|a_{0}\right| \sigma_{0}+\mathbf{r} \cdot \boldsymbol{\sigma} .
$$

By identifying between the two members of Eq. (28), we get

$$
\begin{gathered}
\left|a_{0}\right|^{2}+\|\mathbf{r}\|^{2}= \pm\left|a_{0}\right|, \\
2\left|a_{0}\right| \mathbf{r}=\mathbf{r} .
\end{gathered}
$$

Let us now analyze the various situations coming from these two simultaneous equations. If we take the minus sign in Eq. (29) it follows that

$$
\left|a_{0}\right|=0, \quad\|\mathbf{r}\|=0
$$

i.e., from Eq. (25):

$$
\mathrm{P}=\mathrm{O} \text {, }
$$

where we have labeled $O$ the null operator.

If we want to have $P \neq O$, we should take in Eq. (29) and, consequently in Eq. (25) the plus sign. Further on there are two situations. If $\mathbf{r}=\mathbf{0}$, from Eq. (29) we get $\left|a_{0}\right|=1$, and with Eq. (25):

$$
\mathrm{P}=\sigma_{0} \equiv 1 \text {. }
$$

Finally if $\mathbf{r} \neq \mathbf{0}$, Eq. (30) leads to $\left|a_{0}\right|=\frac{1}{2}$, hence

$$
\mathrm{P}=\frac{1}{2}\left(\sigma_{0}+\mathbf{n} \cdot \boldsymbol{\sigma}\right),
$$

with $\mathbf{n}$ a real unit vector, in accordance with Eq. (1).

If we refer again to the polarization devices, Eq. (34) is the operator of an orthogonal polarizer whose Poincaré axis is $\mathbf{n}$ (evidently, such an operator does not determine a rotation of the state on the Poincaré sphere around its axis, but a spherical projection on its axis).

\section{Symmetries}

A symmetry is a unitary and self-adjoint operator. Note that all the four Pauli matrices are symmetries. Also, S is a symmetry if and only if

$$
\mathrm{S}=2 \mathrm{P}-\mathrm{I},
$$

where $P$ is an orthogonal projection. Thus, if we exclude the extremal cases $P=0$ and $P=I$ from Eq. (34) we get that $S$ is a symmetry if and only if

$$
\mathrm{S}=\mathbf{n} \cdot \boldsymbol{\sigma},
$$

where $\mathbf{n}$ is a real unit vector.

\section{PAULI EXPANSION OF SOME NORMAL AND NONNORMAL DEVICE OPERATORS IN POLARIZATION OPTICS}

The Pauli algebraic forms of various normal device operators, either Hermitian (corresponding to orthogonal polarizers) or unitary (corresponding to retarders), are largely used in polarization optics. ${ }^{1-8,33,34}$ In particular, all the homogeneous canonical optical devices are of the orthogonal kind (orthogonal eigenvectors). ${ }^{25}$

But it is well known ${ }^{18,35,36}$ that for those media that exhibit simultaneously optical activity, linear birefringence, and linear dichroism, the eigenstates are no longer orthogonal. On the other hand, some of the inhomogeneous, composite devices can be nonorthogonal (skew eigenvec- 
tors) even when their components are of the orthogonal kind. ${ }^{26,27}$ The non-Hermitian polarizers have been taken into consideration ${ }^{28}$ in connection with the subject of the general quantum measurement. ${ }^{29-31}$ In spite of all this, the investigations concerning the nonnormal operators remain somewhat peripheral in the polarization theory. In particular no analysis of the Pauli algebraic form of the operators of nonorthogonal polarization devices has been done until now. Therefore, we shall deduce here the Pauli expansions of the (nonnormal, non-Hermitian) operators of some widespread nonorthogonal (skew) polarizers.

\section{A. Nonorthogonal Devices}

The simplest example of a nonnormal device operator in polarization optics is that of a non-Hermitian linear polarizer obtained by sandwiching together two normal linear polarizers at different azimuths $(\theta \neq 0, \pi / 2)$ :

$$
\mathrm{P}=\mathrm{P}_{\left|P_{\theta}\right\rangle} \mathrm{P}_{\left|P_{x}\right\rangle} \cdot
$$

The Poincaré axes of the two Hermitian polarizers $\mathrm{P}_{\left|P_{x}\right\rangle}$ and $\mathrm{P}_{\left|P_{\theta}\right\rangle}$ being

$$
\begin{gathered}
\mathbf{n}_{x}(1,0,0), \\
\mathbf{n}_{\theta}(\cos 2 \theta, \sin 2 \theta, 0),
\end{gathered}
$$

their Pauli expansions are, respectively,

$$
\begin{gathered}
\mathrm{P}_{\left|P_{\theta}\right\rangle}=\frac{1}{2}\left(\sigma_{0}+\sigma_{1}\right), \\
\mathrm{P}_{\left|P_{\theta}\right\rangle}=\frac{1}{2}\left(\sigma_{0}+\sigma_{1} \cos 2 \theta+\sigma_{2} \sin 2 \theta\right) .
\end{gathered}
$$

Hence the Pauli expansion of the composed polarizer [Eq. (37)] is

$$
\begin{aligned}
\mathrm{P}= & \frac{1}{4}\left(\sigma_{0}+\sigma_{1} \cos 2 \theta+\sigma_{2} \sin 2 \theta+\sigma_{1}+\sigma_{0} \cos 2 \theta\right. \\
& \left.-i \sigma_{3} \sin 2 \theta\right),=\frac{1}{2} \cos \theta\left[\left(\sigma_{0}+\sigma_{1}\right) \cos \theta+\left(\sigma_{2}-i \sigma_{3}\right) \sin \theta\right]
\end{aligned}
$$

The Stokes coefficient of $\sigma_{3}$ is $-\pi / 2$ out-of-phase (quadrature) with those of $\sigma_{1}$ and $\sigma_{2}$. The Pauli axis vector of the operator (41) is, apart from a constant,

$$
\mathbf{r}(\cos \theta, \sin \theta,-i \sin \theta) .
$$

It is an irreducible complex vector; the operator $\mathrm{P}$ is a nonnormal one.

Another sandwich that acts as a non-Hermitian linear polarizer is constituted by a linear polarizer followed by a half-wave linear retarder at the azimuth $\theta / 2 \neq 0, \pi$. Such an arrangement is used in the half-shade analyzer in polarimetry. Obviously, the half-wave plate shifts the azimuth of the linearly polarized incident light by $\theta$. The operator of this sandwich is

$$
\mathrm{R}_{\left|P_{\theta / 2}\right\rangle}(\pi) \mathrm{P}_{\left|P_{x}\right\rangle} \cdot
$$

Here the Poincaré axes of the linear polarizer and of the half-wave plate are

$$
\begin{gathered}
\mathbf{n}_{\mathrm{P}}=(1,0,0), \\
\mathbf{n}_{\mathrm{R}}=(\cos \theta, \sin \theta, 0),
\end{gathered}
$$

and the Pauli expansion of their operators may be written

$$
\mathrm{P}_{\left|P_{x}\right\rangle}=\frac{1}{2}\left(\sigma_{0}+\sigma_{1}\right),
$$

$$
\mathrm{R}_{\left|P_{\theta / 2}\right\rangle}(\pi)=i\left(\sigma_{1} \cos \theta+\sigma_{2} \sin \theta\right) .
$$

The composite operator is

$$
\begin{aligned}
& \frac{i}{2}\left(\sigma_{1} \cos \theta+\sigma_{2} \sin \theta\right)\left(\sigma_{0}+\sigma_{1}\right) \\
& \quad=\frac{i}{2}\left[\left(\sigma_{0}+\sigma_{1}\right) \cos \theta+\left(\sigma_{2}-i \sigma_{3}\right) \sin \theta\right],
\end{aligned}
$$

which is very similar to Eq. (41) that is physically justifiable: both operators (37) and (43) give $\theta$-linearly polarized light for any input.

Again the Pauli axis vector of the operator is a complex vector:

$$
\mathbf{r}(\cos \theta, \sin \theta,-i \sin \theta)
$$

the operator is a nonnormal one.

Let us consider now another non-Hermitian polarizer, the circular polarizer obtained by laminating together a linear polarizer and a linear $\pi / 2$ retarder with the transmission direction of the polarizer at $45^{\circ}$ to the proper axes of the retarder,

$$
\mathrm{C}=\mathrm{R}_{\left|P_{x}\right\rangle}(\pi / 2) \mathrm{P}_{\left|P_{45^{\circ}}\right\rangle} \cdot
$$

The Poincaré axes of the linear retarder and of the quarter-wave plate are, respectively,

$$
\begin{aligned}
& \mathbf{n}_{\mathrm{P}}(0,1,0), \\
& \mathbf{n}_{\mathrm{R}}(1,0,0) .
\end{aligned}
$$

Their operators may be written as

$$
\begin{gathered}
\mathrm{P}_{\left|P_{45^{\circ}}\right\rangle}=\frac{1}{2}\left(\sigma_{0}+\sigma_{2}\right), \\
\mathrm{R}_{\left|P_{x}\right\rangle}(\pi / 2)=\frac{1}{\sqrt{2}}\left(\sigma_{0}+i \sigma_{1}\right),
\end{gathered}
$$

so that the Pauli expansion of the inhomogeneous circular polarizer operator [Eq. (48)] is

$$
\mathrm{C}=\frac{1}{2 \sqrt{2}}\left(\sigma_{0}+i \sigma_{1}+\sigma_{2}-\sigma_{3}\right) .
$$

The Stokes coefficients of $\sigma_{1}, \sigma_{2}$, and $\sigma_{3}$ are not all in phase nor in opposition. The Pauli axis vector of the operator is complex: 


$$
\mathbf{r}(i, 1,-1)
$$

The operator is not a normal one. This circular polarizer is a non-Hermitian polarizer.

\section{B. Orthogonal Composite Devices}

We shall deduce now, for comparison, the Pauli algebraic expansions of two widespread orthogonal composite devices: the transcendent retarder and the Pancharatnam's $\mathcal{Q H} \mathcal{Q}$ variable retarder.

It is well known that a succession of two half-wave plates at a relative azimuth of $45^{\circ}$ of their axes acts as a $\pi$ circular retarder. This is the simplest transcendent retarder. ${ }^{37}$ A generalization of this device was given by $\mathrm{Ri}$ chartz and $\mathrm{Hsü}^{38}$ by putting the two half-wave plates at a relative azimuth $\theta$,

$$
\mathrm{R}_{T}=\mathrm{R}_{\left|P_{\theta}\right\rangle}(\pi) \mathrm{R}_{\left|P_{x}\right\rangle}(\pi)
$$

The Poincaré axes of the two half-wave plates are

$$
\mathbf{n}(1,0,0),
$$

$$
\mathbf{n}(\cos 2 \theta, \sin 2 \theta, 0),
$$

so that their operators take on the form

$$
\begin{gathered}
\mathrm{R}_{\left|P_{x}\right\rangle}(\pi)=i \sigma_{1}, \\
\mathrm{R}_{\left|P_{\theta}\right\rangle}(\pi)=i\left(\sigma_{1} \cos 2 \theta+\sigma_{2} \sin 2 \theta\right) .
\end{gathered}
$$

Thus, the Pauli expansion of the transcendent retarder [Eq. (53)] is

$$
\mathrm{R}_{T}=-\left(\sigma_{1}^{2} \cos 2 \theta+\sigma_{2} \sigma_{1} \sin 2 \theta\right)=-\left(\mathrm{I} \cos 2 \theta-i \sigma_{3} \sin 2 \theta\right) .
$$

This is a unitary operator with the Poincaré axis

$$
\mathbf{n}(0,0,+1)
$$

and that gives a $-4 \theta$ rotation of the incident state of polarization on the Poincaré sphere about the $|R\rangle$ axis. Hence it corresponds to an (orthogonal) circular retarder:

$$
\mathrm{R}_{T}=e^{\mathrm{i} \pi} \mathrm{R}_{|R\rangle}(-4 \theta) .
$$

By analyzing a more general combination of three birefringent plates, Pancharatnam ${ }^{39}$ noted that a combination of two quarter-wave plates with parallel principal axes, between which is placed a half-wave plate with a variable azimuth of the principal axis, gives rise to a variable linear retarder:

$$
\mathrm{R}_{P}=\mathrm{R}_{\left|P_{x}\right\rangle}(\pi / 2) \mathrm{R}_{\left|P_{\theta}\right\rangle}(\pi) \mathrm{R}_{\left|p_{x}\right\rangle}(\pi / 2) .
$$

Let us express first the $\mathrm{R}_{\left|P_{x}\right\rangle}(\pi / 2)$ quarter-wave plates operators in a Pauli algebraic form. The Poincaré axis of the operators is

$$
\mathbf{n}(1,0,0) \text {, }
$$

and the angle of the rotation induced by these operators on the Poincaré sphere is $\delta=\pi / 2$. Hence with Eq. (2) the operators may be written,

$$
\mathrm{R}_{\left|P_{x}\right\rangle}(\pi)=\frac{1}{\sqrt{2}}\left(\mathrm{I}+i \sigma_{1}\right) .
$$

Similarly, for the half-wave plate of the $\theta$-fast axis, $\mathrm{R}_{\left|P_{\theta}\right\rangle}(\pi)$, we have

$$
\mathbf{n}(\cos 2 \theta, \sin 2 \theta, 0),
$$

and $\delta=\pi$. The Pauli algebraic expression of its operator is

$$
\mathrm{R}_{\left|P_{\theta}\right\rangle}(\pi)=i\left(\sigma_{1} \cos 2 \theta+\sigma_{2} \sin 2 \theta\right) .
$$

With Eqs. (64) and (66) in Eq. (62) we get

$$
\begin{gathered}
\mathrm{R}_{P}=\frac{i}{2}\left(\sigma_{1} \cos 2 \theta+\sigma_{2} \sin 2 \theta+i \mid \cos 2 \theta+\sigma_{3} \sin 2 \theta\right. \\
\left.+i \mid \cos 2 \theta-\sigma_{3} \sin 2 \theta-\sigma_{1} \cos 2 \theta+\sigma_{2} \sin 2 \theta\right), \\
\mathrm{R}_{P}=-\left(\mid \cos 2 \theta-i \sigma_{2} \sin 2 \theta\right) .
\end{gathered}
$$

Apart from a phase factor $e^{\mathrm{i} \pi}=-1$, Eq. (67) has the form of a unitary operator, with the Poincaré axis

$$
\mathbf{n}(0,-1,0) \text {, }
$$

and inducing a rotation $\delta=4 \theta$ on the Poincaré sphere.

This is a $4 \theta$ linear retarder of fast-axis $-45^{\circ}$ (or a $-4 \theta$ linear retarder of fast-axis $45^{\circ}$ ):

$$
\mathrm{R}_{\left|P_{x}\right\rangle}(\pi / 2) \mathrm{R}_{\left|P_{\theta}\right\rangle}(\pi) \mathrm{R}_{\left|P_{x}\right\rangle}(\pi / 2)=\mathrm{R}_{\left|P_{45^{\circ}}\right\rangle}(-4 \theta) \equiv \mathrm{R}_{\left|P_{-45^{\circ}}\right\rangle}(4 \theta) .
$$

From the main viewpoint of our analysis we have to point out that the Pauli axes of these last two normal (unitary) operators, Eqs. (60) and (68), reduce to their real, Poincaré axes on the Poincaré sphere.

\section{CONCLUSIONS}

The Pauli expansions of the orthogonal projector [Eq. (1)] and of the unitary operator [Eq. (2)] are currently handled as device operators of the canonical ideal polarizers and retarders, respectively, in polarization optics, where these expansions have been established inductively, on an experimental ground.

Generally, the polarization devices are not of the orthogonal kind. The eigenvectors of propagating light in crystals are, generally (and naturally), nonorthogonal. Nonnormal operators govern the light propagation in crystals, particularly in polarization devices, in the most general case. This is one of the reasons why the interest in nonnormal operators in physics is growing. ${ }^{14-23}$

Starting from this very definite interest, we have given a unified theory of the Pauli algebraic development of various normal and nonnormal operators. In this framework, the Pauli expressions of the orthogonal projectors and unitary operators are obtained in a hierarchic deductive way, together with other kinds of normal and nonnormal operators.

In this approach, to each operator defined on a complex vector space of two dimensions over the field of complex numbers $\mathbf{C}^{1}$ corresponds a vector in $\mathbf{C}^{3}$ that we call the Pauli axis of the operator. In particular, for normal operators their Pauli axes are real vectors or are reducible by a 
phase shift to real vectors, whereas the Pauli axes of the nonnormal operators are irreducible complex vectors.

Bearing in mind the great interest manifested in recent years, in a large variety of physical problems, for nonnormal operators (defined as "non-Hermitian" or "nonunitary" and handled especially in matrix forms), we hope that our results will be useful in the mathematical approach to a larger field of physical problems.

Well known, especially in the past decade, the group theory of $\mathrm{SL}(2, c)$ and of some of its subgroups was extensively applied in various fields of classical and quantum optics, e.g., ray optics, ${ }^{40}$ beam propagation through firstorder systems, ${ }^{41}$ analysis of the states of light with orbital angular momentum, ${ }^{42}$ polarization optics, ${ }^{43}$ multilayer optics, ${ }^{44,45}$ interferometry, ${ }^{46}$ and coherent and squeezed states of light. ${ }^{47}$ Bearing in mind that $\operatorname{SL}(2, c)$ is locally isomorphic to the six-parameter Lorentz group $\mathrm{SO}(3,1)$, a physical system that can be analyzed in terms of $\mathrm{SL}(2, c)$ language can be equally explained in the language of the Lorentz group. ${ }^{48}$

From the viewpoint of the group theory, the nonHermitian polarizer [Eq. (37)] is an example of Wigner rotation: The two Hermitian polarizers of a sandwich such as Eq. (37) are described by squeeze operators, and their product is not a squeeze operator, but a squeeze operator followed or preceded by a rotation.

We think that, generally, the group-theoretical approach has not, until now, paid special attention to the dichotomy of normal and nonnormal operators, perhaps because the normal operators-although a very important class-do not form a group. This problem has appeared from another field of interest in physics, ${ }^{14-31}$ and probably the group-theoretical approach will have an important word to say in this concern.

\section{ACKNOWLEDGMENTS}

We thank the reviewers for comments that resulted in the substantial improvement of the manuscript.

The authors' email addresses are ttudor@ifin.nipne.ro and aurelian@fen.bilkent.edu.tr.

\section{REFERENCES}

1. M. V. Berry and S. Klein, "Geometric phases from stacks of crystal plates,” J. Mod. Opt. 43, 165-180 (1996).

2. S. F. Li, "Jones-matrix analysis with Pauli matrices: application to ellipsometry," J. Opt. Soc. Am. A 17, 920-926 (2000).

3. C. Whitney, "Pauli-algebraic operators in polarization optics," J. Opt. Soc. Am. 61, 1207-1213 (1971).

4. R. Bhandari, "Halfwave retarder for all polarization states," Appl. Opt. 36, 2799-2801 (1997).

5. R. Bhandari and G. D. Love, "Polarization eigenmodes of a QHQ retarder - some new features," Opt. Commun. 110, 479-484 (1994).

6. R. Bhandari, "Interferometry without beam splitters - a sensitive technique for spinor phases," Phys. Lett. A 180, 21-24 (1993).

7. R. Bhandari, "Observation of Dirac singularities with light polarization. II," Phys. Lett. A 171, 267-270 (1992).

8. R. Bhandari, "Evolution of light beams in polarization and direction," Phys. Lett. B 175, 111-122 (1991).

9. C. Cohen-Tannoudji, B. Diu, and F. Laloë, Quantum Mechanics (Wiley, 1997), Vol. 1.
10. R. P. Feynman, R. B. Leighton, and M. Sands, The Feynman Lectures on Physics III (Quantum Mechanics) (Addison-Wesley, 1965).

11. S. M. Barnett and P. M. Radmore, Methods in Theoretical Quantum Optics (Clarendon, 2003)

12. R. M. A. Azzam and N. M. Bashara, Ellipsometry and Polarized Light (Elsevier, 1996).

13. M. A. Nielsen and I. L. Chuang, Quantum Computation and Quantum Information (Cambridge U. Press, 2002).

14. M. V. Berry, "Physics of nonhermitian degeneracies," Czech. J. Phys. 55, 1039-1046 (2004).

15. W. D. Heiss, "Exceptional points - their universal occurrence and their physical significance," Czech. J. Phys. 55, 1091-1099 (2004).

16. A. P. Seyranian, O. N. Kirillov, and A. A. Mailybaev, "Coupling of eigenvalues of complex matrices at diabolic and exceptional points," J. Phys. A 38, 1723-1740 (2005).

17. M. V. Berry, "Mode degeneracies and the Petermann excess-noise factor for unstable lasers," J. Mod. Opt. 50, 63-81 (2003).

18. M. V. Berry and M. R. Dennis, "The optical singularities of birefringent dichroic chiral crystals," Proc. R. Soc. London, Ser. A 459, 1261-1292 (2003).

19. M. V. Berry and D. H. J. O'Dell, "Diffraction by volume gratings with imaginary potentials," J. Phys. A 31, 2093-2101 (1998).

20. W. D. Heiss, M. Müller, and I. Rotter, "Collectivity, phase transitions, and exceptional points in open quantum systems," Phys. Rev. E 58, 2894-2901 (1998).

21. T. Stehmann, W. D. Heiss, and F. G. Scholtz, "Observation of exceptional points in electronic circuits," J. Phys. A 37, 7813-7819 (2004)

22. M. Philipp, P. von Brentano, G. Pascovici, and A. Richter, "Frequency and width crossing of two interacting resonances in a microwave cavity," Phys. Rev. E 62, 1922-1926 (2000).

23. W. D. Heiss, "Repulsion of resonant states and exceptional points," Phys. Rev. E 61, 929-932 (2000).

24. F. Keck, H. J. Korsch, and S. Mossmann, "Unfolding a diabolic point: a generalized crossing scenario," J. Phys. A 36, 2125-2137 (2003).

25. T. Tudor, "Operatorial form of the theory of polarization optical devices: I. Spectral theory of the basic devices," Optik (Stuttgart) 114, 539-547 (2003).

26. T. Tudor, "Operatorial form of the theory of polarization optical devices: II. Spectral theory of the composite devices," Optik (Stuttgart) 115, 173-180 (2004).

27. S.-Y. Lu and R. A. Chipman, "Homogeneous and inhomogeneous Jones matrices," J. Opt. Soc. Am. A 11, 766-773 (1994).

28. T. Tudor, "Generalized observables in polarization optics," J. Phys. A 36, 9567-9590 (2003).

29. E. B. Davies and J. T. Lewis, "An operatorial approach to quantum probability," Commun. Math. Phys. 17, 239-260 (1970).

30. P. Busch, P. J. Lathi, and P. Mittelstaedt, The Quantum Theory of Measurement (Springer, 1996).

31. M. de Muynck, "An alternative to the Lüders generalization of the von Neumann projection and its interpretation," J. Phys. A 31, 431-444 (1998).

32. Ch. Brosseau, Fundamentals of Polarized Light (Wiley, 1998).

33. R. Simon and N. Mukunda, "Universal SU(2) gadget for polarization optics," Phys. Lett. A 138, 474-480 (1989).

34. A. S. Marathay, "Operator formalism in the theory of partial polarization," J. Opt. Soc. Am. 55, 969-980 (1965).

35. S. Pancharatnam, "The propagation of light in absorbing biaxial crystals. Part I. Theoretical," Proc. Indian Acad. Sci., Sect. A 42, 86-109 (1955).

36. S. Pancharatnam, Collected Works of S. Pancharatnam (Oxford U. Press, 1975).

37. W. A. Shurcliff, Polarized Light (Harvard U. Press, 1962).

38. M. Richartz, H.-Y. Hsü, "Analysis of elliptical polarization," J. Opt. Soc. Am. 39, 136-157 (1949).

39. S. Pancharatnam, "Achromatic combinations of 
birefringent plates. Part II: An achromatic quarter-wave plate," Proc. Indian Acad. Sci., Sect. A 41, 137-144 (1955).

40. S. Baskal, E. Georgieva, Y. S. Kim, and M. E. Noz, "Lorentz group in classical ray optics," J. Opt. B: Quantum Semiclassical Opt. 6, S455-S472 (2004).

41. R. Simon and N. Mukunda, "Iwasawa decomposition in first-order optics: universal treatment of shape-invariant propagation for coherent and partially coherent beams," J. Opt. Soc. Am. A 15, 2147-2155 (1998).

42. G. S. Agarwal, "SU(2) structure of the Poincaré sphere for light beams with orbital angular momentum," J. Opt. Soc. Am. A 16, 2914-2916 (1999).

43. D. Han, Y. S. Kim, and M. E. Noz, "Jones-matrix formalism as a representation of the Lorentz group," J. Opt. Soc. Am. A 14, 2290-2298 (1997).
44. E. Georgieva, "Slide-rule-like property of Wigner's little groups and cyclic $\mathrm{S}$ matrices for multilayer optics," Phys. Rev. E 68, 026606 (2003).

45. J. J. Monzón and L. L. Sanchez-Soto, "Multilayer optics as an analog computer for testing special relativity," Phys. Lett. A 262, 18-26 (1999).

46. D. Han, Y. S. Kim, and M. E. Noz, "Interferometers and decoherence matrices," Phys. Rev. E 61, 5907-5913 (2000).

47. D. Han, Y. S. Kim, and M. E. Noz, "Linear canonical transformation of coherent and squeezed states in the Wigner phase space," Phys. Rev. A 37, 807-814 (1988).

48. S. Baskal and Y. S. Kim, "The language of Einstein spoken by optical instruments," Opt. Spectrosc. 99, 443-446 (2005). 